\title{
Strength resistance of reinforced concrete elements of high-rise buildings under dynamic loads
}

\author{
Mikhail Berlinov ${ }^{{ }^{*}}$ \\ ${ }^{1}$ Moscow State Construction University (National Research University), 129337, Moscow, Yaroslavl \\ highway, d. 26, Russian Federation
}

\begin{abstract}
A new method for calculating reinforced concrete constructions of high-rise buildings under dynamic loads from wind, seismic, transport and equipment based on the initial assumptions of the modern phenomenological theory of a nonlinearly deformable elastic-creeping body is proposed. In the article examined the influence of reinforcement on the work of concrete in the conditions of triaxial stress-strain state, based on the compatibility of the deformation of concrete and reinforcement. Mathematical phenomenological equations have been obtained that make it possible to calculate the reinforced concrete elements working without and with cracks. A method for linearizing of these equations based on integral estimates is proposed, which provides the fixation of the vibro-creep processes in the considered period of time. Application of such a technique using the finite-difference method, step method and successive approximations will allow to find a numerical solution of the problem. Such an approach in the design of reinforced concrete constructions will allow not only more fully to take into account the real conditions of their work, revealing additional reserves of load capacity, but also to open additional opportunities for analysis and forecasting their functioning at various stages of operation.
\end{abstract}

\section{Introduction}

Increasing efficiency and quality of high-rise construction under conditions of dynamic loads from wind, seismic, traffic and process loads requires the use of more economical and reliable constructions, which becomes possible if the advanced methods of calculation are used in the design [1]. The creation of such methods is associated with the need to more fully reflect the real properties of materials accounting their actual behavior under the influence of external loads. Development of such methods of calculation is closely linked with the consideration of the nonlinear and rheological properties of materials [2].

It is known that the technique becomes all the more perfect, the more it links the object with the surrounding real world, but it is known from philosophy that the number of such

\footnotetext{
* Corresponding author: berlinov2010@mail.ru
} 
links is infinite, but allowing for a sufficient majority of the basic relationships will help to solve this problem [3].

Obviously, the improvement of computational methods lies in the ways of their increasing complexity due to the necessity of using a very complex mathematical apparatus, since it is this factor that can realize the aforementioned variety of connections [4], and the use at the present stage of the development of society. And the use of such a powerful tool as modern computers and their software allows you to implement fairly complex mathematical solutions with the help of numerical methods and the necessary linearization of nonlinear dependencies [5].

\section{Results section}

For dynamic calculation, as a rule, well-known equations of dynamic equilibrium are used [6], which have the following form:

$$
\begin{aligned}
& \frac{\partial \sigma_{x}}{\partial x}+\frac{\partial \tau_{x y}}{\partial y}+\frac{\partial \tau_{x z}}{\partial z}+X=\rho \frac{\partial^{2} u}{\partial t^{2}} \\
& \left.\frac{\partial \tau_{y x}}{\partial x}+\frac{\partial \sigma_{y}}{\partial y}+\frac{\partial \tau_{y z}}{\partial z}+Y=\rho \frac{\partial^{2} v}{\partial t^{2}}\right\} \\
& \frac{\partial \tau_{z x}}{\partial x}+\frac{\partial \tau_{z y}}{\partial y}+\frac{\partial \sigma_{z}}{\partial z}+Z=\rho \frac{\partial^{2} w}{\partial t^{2}}
\end{aligned}
$$

where: $\mathrm{X}, \mathrm{Y}$, and $\mathrm{Z}$ are the projections of the volume forces on the corresponding axes; $\rho$ is the mass of the material; $\mathrm{u}, \mathrm{v}$, and $\mathrm{w}$ displacement along the Cartesian coordinate axes, t-time.

The proposed calculation technique is based on the main assumptions of the modern phenomenological theory of a nonlinearly deformable elastic-creeping body, and takes into account the geometric nonlinearity expressed in nonlinear dependencies between strains and stresses (the Cauchy conjecture is regarded as usually fair). Therefore, the resolution of these equations, taking into account all these factors, will be substantially complicated.

For the derivation of resolving mathematical dependencies, we use a well-known technique that allows us to obtain resolving equations in stresses:

$$
\begin{aligned}
& \Theta(t)=\frac{1-2 v}{E_{0}(t)} S_{m}(F) F(t)-(1-2 v) S_{p}(F) F(t) K\left(F, \omega, \omega_{\min }, t\right) C\left(t, t_{0}\right) \\
& -(1-2 v) \int_{t_{0}}^{t} S_{n}(F) F(\tau) K\left(F, \omega, \omega_{\min ,} \tau\right) \frac{\partial}{\partial d \tau} C(t . \tau) d \tau
\end{aligned}
$$

here: $v$ - coefficient of relative transverse deformations; $E_{0}(t)$ is the modulus of elasticinstantaneous deformations; $\Theta=\varepsilon_{\mathrm{x}}(\mathrm{t})+\varepsilon_{\mathrm{y}}(\mathrm{t})+\varepsilon_{\mathrm{z}}(\mathrm{t})$ is the relative volume deformation;

$\mathrm{K}\left(\sigma_{\mathrm{x}}, \omega, \omega_{\min }, \tau\right)$ is the vibro-creep coefficient; $\mathrm{C}\left(\mathrm{t}, \mathrm{t}_{0}\right)$ is a measure of creep strains.

The nonlinearity functions of elastic-instantaneous deformations and creep strains have the form:

$$
\begin{aligned}
& \mathrm{S}_{\mathrm{m}}(\mathrm{F}) \mathrm{F}(\mathrm{t})=\sigma_{\mathrm{x}}(\mathrm{t}) \mathrm{S}_{\mathrm{m}}\left[\sigma_{\mathrm{x}}(\mathrm{t}) / \mathrm{R}(\rho, \mathrm{t})\right]+\sigma_{\mathrm{y}}(\mathrm{t}) \mathrm{S}_{\mathrm{m}}\left[\sigma_{\mathrm{y}}(\mathrm{t}) / \mathrm{R}(\rho, \mathrm{t})\right]+\sigma_{\mathrm{z}}(\mathrm{t}) \mathrm{S}_{\mathrm{m}}\left[\sigma_{\mathrm{z}}(\mathrm{t}) / \mathrm{R}(\rho, \mathrm{t})\right] ; \\
& \mathrm{S}_{\mathrm{p}}(\mathrm{F}) \mathrm{F}(\tau) \mathrm{K}\left(\mathrm{F}, \omega, \omega_{\min }, \tau\right)=\sigma_{\mathrm{x}}(\tau) \mathrm{S}_{\mathrm{p}}\left[\sigma_{\mathrm{x}}(\tau) / \mathrm{R}(\rho, \tau)\right] \mathrm{K}\left(\sigma_{\mathrm{x}}, \omega, \omega_{\min }, \tau\right)+ \\
& +\sigma_{\mathrm{y}}(\tau) \mathrm{S}_{\mathrm{p}}\left[\sigma_{\mathrm{y}}(\tau) / \mathrm{R}(\rho, \tau)\right] K\left(\sigma_{\mathrm{y}}, \omega, \omega_{\min }, \tau\right)+\sigma_{\mathrm{z}}(\tau) \mathrm{S}_{\mathrm{p}}\left[\sigma_{\mathrm{z}}(\tau) / \mathrm{R}(\rho, \tau)\right] K\left(\sigma_{\mathrm{z}}, \omega, \omega_{\min }, \tau\right)
\end{aligned}
$$

here: $S_{m}\left[\sigma_{x}(t) / R(\rho, t)\right]$ is the nonlinearity function for uniaxial deformation; $R(\rho, t)$ concrete strength under uniaxial dynamic deformation; $\rho$ - coefficient of asymmetry of the cycle. 
If the connection between stresses and strains is taken in a known inverse form, which in the case of a uniaxial stress-strain state has the form:

$$
\begin{gathered}
\sigma_{x}(t)=E_{0}(t) \varepsilon_{x}(t) \varphi_{m}\left[\varepsilon_{x}(t)\right]+Q\left(t, t_{0}\right) \varepsilon_{x}(t) \varphi_{p}\left[\varepsilon_{x}(t)\right]-\int_{t_{0}}^{t} \frac{\varepsilon_{x}(\tau) \varphi_{p}\left[\varepsilon_{x}(\tau)\right]}{K\left(\sigma_{x}, \omega_{\min }, \omega, \tau\right)} \frac{\partial}{\partial \tau} Q(t, \tau) d \tau, \\
\text { where: } \varphi_{m}=1+\eta_{m}\left(\frac{\varepsilon}{\varepsilon_{R}}\right)^{a_{m}} \text { and } \varphi_{p}=1+\eta_{p}\left(\frac{\varepsilon}{\varepsilon_{R}}\right)^{a_{p}}
\end{gathered}
$$

- functions of nonlinearity of deformations; $\mathrm{Q}\left(\mathrm{t}, \mathrm{t}_{0}\right)$ - the hereditary function of the second kind

here: $\varepsilon_{R}$ - is the limiting relative deformation of a given class of concrete, at the moment immediately preceding the failure, with acceptable accuracy, determined from experimental data or analytically by the formula:

$$
\varepsilon_{R}=\frac{R(\rho, t)}{E(t)}
$$

here: $\mathrm{E}(\mathrm{t})$ is the elasto-plastic modulus of concrete deformations

It should be noted that the coefficients of dependences (5) can be obtained directly from the real diagram $(\sigma-\varepsilon)$ or by solving logarithmic equations systems mathematically.

For tangential stresses, the formula takes the form:

$$
\begin{aligned}
& \tau_{x y}(t)=\frac{E_{m}^{0}(t)}{2(1+v)} \gamma_{x y}(t) \varphi_{m}\left[\gamma_{x y}(t)\right]+\frac{Q\left(t, t_{0}\right)}{(1+v)} \gamma_{x y}(t) \varphi_{p} \times \\
& \times\left[\gamma_{x y}(t)\right]-\frac{1}{2(1+v)} \int_{t_{0}}^{t} \frac{\gamma_{x y}(\tau) \varphi_{p}\left[\gamma_{x y}(\tau)\right]}{K\left(\sigma_{x}, \omega_{\min }, \omega, \tau\right)} \frac{\partial}{\partial \tau} Q(t, \tau) d \tau .
\end{aligned}
$$

The values of the characteristics entering into equation (7) can be found similarly to (4)

The hereditary function of the second kind in the dependences (4) and (7) can be found analytically by the generally accepted method from the solution of the following integral equation:

$$
\left.L(t, \tau)-Q(t, \tau)=\int_{t_{0}}^{t} L(\xi, \tau) Q(t, \xi) d \xi \quad\right\}
$$

where: $\quad L(t, \tau)=-E(\tau) \frac{\partial}{\partial \tau}\left[\frac{1}{E(\tau)}+C(t, \tau)\right]$.

The relationship between stresses and deformations for an elastic-creeping body is established by considering this expression together with equation (2), from which we obtain:

$$
\begin{aligned}
& \sigma_{x}(t)=\frac{v E_{0}(t)}{(1+v)(1-2 v)} \varphi_{m}(\Theta) \Theta(t)+\frac{v Q\left(t, t_{0}\right)}{(1+v)(1-2 v)} \varphi_{p}(\Theta) \Theta(t)-\frac{v}{(1+v)(1-2 v)} \int_{t_{0}}^{t} \frac{\varphi_{n}(\Theta) \Theta(\tau)}{K\left(F, \omega_{\min }, \omega, \tau\right)} . \\
& \cdot \frac{\partial}{\partial \tau} Q(t, \tau) d \tau+\frac{E_{0}(t)}{2(1+v)} \varepsilon_{x}(t) \varphi_{m}\left(\varepsilon_{x}\right)-\frac{1}{2(1+v)} \int_{t_{0}}^{t} \frac{\varepsilon_{x}(\tau) \varphi_{n}\left(\varepsilon_{x}\right)}{K\left(\sigma_{x}, \omega_{\min }, \omega, \tau\right)} \frac{\partial}{\partial \tau} Q(t, \tau) d \tau
\end{aligned}
$$

where: analogous to $(3), \varphi_{m}(\Theta)$ is a unified function of nonlinearity determined on the basis of experimental data or analytically:

$$
\varphi_{\mathrm{m}}(\Theta) \Theta(\mathrm{t})=\varepsilon_{\mathrm{x}}(\mathrm{t}) \varphi_{\mathrm{m}}\left[\varepsilon_{\mathrm{x}}(\mathrm{t}) / \varepsilon_{\mathrm{R}}(\mathrm{t})\right]+\varepsilon_{\mathrm{y}}(\mathrm{t}) \varphi_{\mathrm{m}}\left[\varepsilon_{\mathrm{y}}(\mathrm{t}) / \varepsilon_{\mathrm{R}}(\mathrm{t})\right]+\varepsilon_{\mathrm{z}}(\mathrm{t}) \varphi_{\mathrm{m}}\left[\varepsilon_{\mathrm{z}}(\mathrm{t}) / \varepsilon_{\mathrm{R}}(\mathrm{t})\right]
$$


For the case of quasilinear creep, formula (9) can be somewhat simplified to a more acceptable form:

$$
\begin{aligned}
& \sigma_{x}(t)=\Theta\left[\frac{\varepsilon(t)}{\varepsilon_{R}(t)}\right]\left\{\frac{v E_{0}(t)}{(1+v)(1-2 v)} \Theta(t)+\frac{v Q\left(t, t_{0}\right) \Theta(t)}{(1+v)(1-2 v)}-\frac{v}{(1+v)(1-2 v)} \int_{t_{0}}^{t} \frac{\Theta(\tau)}{K\left(F, \omega_{\min }, \omega, \tau\right)} .\right. \\
& \left.\cdot \frac{\partial}{\partial \tau} Q(t, \tau) d \tau+\frac{E_{0}(t)}{2(1+v)} \varepsilon_{x}(t)-\frac{1}{2(1+v)} \int_{t_{0}}^{t} \frac{\varepsilon_{x}(\tau)}{K\left(\sigma_{x}, \omega_{\min }, \omega, \tau\right)} \frac{\partial}{\partial \tau} Q(t, \tau) d \tau\right\}
\end{aligned}
$$

To reduce the writing, equations (9) and (11) can be represented as:

$$
\sigma_{x}(t)=\tilde{\lambda}(t) \Theta(t)+2 \widetilde{\mu}(t) \varepsilon_{x}(t)
$$

where: $\tilde{\lambda}(t)$ and $\tilde{\mu}(t)$ are respectively non-linear integral operators.

The dependences for tangential stresses taking into account (8) take the form:

$$
\begin{aligned}
\tau_{x y}= & \frac{E_{0}(t)}{2(1+v)} \gamma_{x y}(t) S_{m}\left[\frac{\gamma_{x y}(t)}{\gamma_{R}(\rho, t)}\right]+\frac{Q\left(t, t_{0}\right)}{2(1+v)} \gamma_{x y} S_{p}\left[\frac{\gamma_{x y}(t)}{\gamma_{R}(\rho, t)}\right]- \\
& \left.-\frac{1}{2(1+v)} \int_{t_{0}}^{t} \gamma_{x y}(\tau) S_{n}\left[\frac{\gamma_{x y}(\tau)}{\gamma_{R}(\rho, \tau)}\right] \frac{1}{K\left(\tau_{x y}, \omega_{\min }, \omega, \tau\right)} \frac{\partial}{\partial \tau} Q(t, \tau) d \tau\right\}
\end{aligned}
$$

By resorting to similar arguments, one can obtain analogous relations for $\sigma_{y}(t), \sigma_{z}(t)$, $\tau_{\mathrm{yz}}(\mathrm{t})$ and $\tau_{\mathrm{zx}}(\mathrm{t})$, which can finally be represented in the following form:

$$
\begin{array}{rrr}
\sigma_{x}(t)=\tilde{\lambda}(t) \Theta(t)+2 \widetilde{\mu}(t) \varepsilon_{x}(t) ; & \tau_{x y}=\widetilde{\mu}(t) \gamma_{x y}(t) \\
\sigma_{y}(t)=\tilde{\lambda}(t) \Theta(t)+2 \widetilde{\mu}(t) \varepsilon_{y}(t) ; & \tau_{y z}=\widetilde{\mu}(t) \gamma_{y z}(t) \\
\sigma_{z}(t)=\tilde{\lambda}(t) \Theta(t)+2 \widetilde{\mu}(t) \varepsilon_{z}(t) ; & \tau_{z x}=\widetilde{\mu}(t) \gamma_{z x}(t)
\end{array}
$$

Analyzing equations (14), we can conclude that the rejection of nonlinearity and rheology of deformation will turn them into mathematical expressions of the inverse form of Hooke's law.

Considering the compatibility of deformation of concrete and reinforcement and carrying out similar reasoning, it is possible to easily obtain solving equations for reinforced elements in orthotropic reinforcement (Figure 1.a), working both with cracks and without them. For example, for elements operating without cracks, the integral operators will have a slightly different form than in (14), since they will take into account the reinforcement of the element, namely:

$$
\begin{aligned}
& \hat{\lambda}_{i}(t)=\frac{v E_{m}^{0}(t)\left(1-\mu_{i}\right)\left(1+\alpha \mu_{i}\right)}{(1+v)(1-2 v)} \cdots- \\
& \left.-\frac{v\left(1-\mu_{i}\right)}{(1+v)(1-2 v)} \int_{t_{0}}^{t} \frac{\ldots}{1+\mu_{i} E_{s} K\left(F, \omega_{\min }, \omega, \tau\right)} \frac{\partial}{\partial \tau} Q(t, \tau) d \tau\right] \\
& \hat{\mu}_{i}(t)=\frac{E_{m}^{0}(t)\left(1+\alpha \mu_{i}\right)}{2(1+v)} \cdots-\frac{1}{2(1+v)} \int_{t_{0}}^{t} \frac{\ldots}{1+\mu_{i} E_{s} K\left(\sigma_{x}, \omega_{\min }, \omega, \tau\right)} \frac{\partial}{\partial \tau} Q(t, \tau) d \tau
\end{aligned}
$$

The proposed method is, in principle, allows to consider any direction of reinforcement, such as an inclined $A_{i}$ (Figure 1,b). In this case, the reinforcement ratio: $\mu_{s i}=A_{s i} / a_{1} a_{2} ; a_{1}$ 
and $\mathrm{a}_{2}$ - the distance between the individual rods with oblique reinforcement. Note that in this expression ratio $\alpha_{\mathrm{c}}=\mathrm{K}\left(\sigma_{\mathrm{bp}}, \omega_{\min }, \omega, \tau\right) \mathrm{C}(\mathrm{t}, \tau) / \mathrm{K}_{\mathrm{s}}\left(\sigma_{\mathrm{sp}}, \omega_{\min }, \omega, \tau\right) \mathrm{C}_{\mathrm{s}}(\mathrm{t}, \tau)$ can be considered as reinforcement reduction coefficient for concrete, interpreted with respect to the creep deformation in contrast to the values of $\alpha=E_{s} / E_{m}^{0}(t)$ valid for elastic-instantaneous deformations.

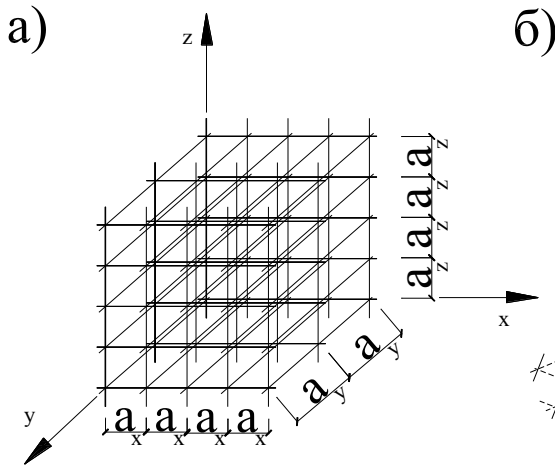

б)

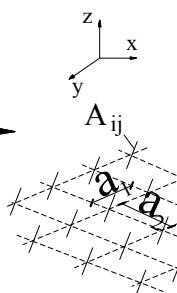

Fig.1. Directions of reinforcement; a) - is orthotropic; b) - inclined.

Omitting even more obvious arguments for elements working with cracks without taking into account the nonlinearity and rheology of deformation of reinforcing steel in all three directions of the coordinate axes, we obtain:

$$
\begin{aligned}
& \left.\hat{\lambda}_{i}(t)=\frac{v E_{s}^{0}(t) \psi_{s i} \mu_{i}}{\left(1+v_{s}\right)\left(1-2 v_{s}\right)} \cdots-\frac{v \psi_{s i} \mu_{i}}{\left(1+v_{s}\right)\left(1-2 v_{s}\right)} \int_{t_{0}}^{t} \cdots \frac{\partial}{\partial \tau} Q_{s}(t, \tau) d \tau\right] \\
& \hat{\mu}_{i}(t)=\frac{E_{s}^{0}(t) \psi_{s i} n_{t i} \mu_{s i}}{2(1+v)} \cdots-\frac{\psi_{s i} n_{t i} \mu_{s i}}{2(1+v)} \int_{t_{0}}^{t} \cdots \frac{\partial}{\partial \tau} Q_{s}(t, \tau) d \tau
\end{aligned}
$$

Where the integral operators turn into physical constants for the deformation of the reinforcement, really taking into account the work of concrete on the sections between the cracks. Those, in the final analysis, we obtain three resolving systems of equations differing only in the values of integral operators, which take into account the reinforcement and appearance of cracks.

Obviously, taking into account the dependences (14), (15) and (16) when searching for a numerical solution, with the aid of some calculating apparatus (the finite element method, for example), will encounter insurmountable mathematical difficulties, since it will require closed integration of the resolving equations; therefore, in order to obtain engineering solutions in numerical form, it is necessary to linearize the problem at least in the physical area. This linearization is possible on the basis of the well-known method of integral estimates.

\section{Discussion}

Calculation of reinforced concrete constructions in the operational state according to the accepted design standards is usually performed with the help of the well-known linear apparatus of the theory of elasticity or structural mechanics, and in the classical theory nonlinearity, rheology and anisotropy of deformation are ignored. These imperfections of materials are estimated only by multiplying the calculated characteristics by some empirical coefficients that do not depend on the modes of action of external loads and, what is 
especially important, the duration of their action. More advanced engineering methods that have become popular recently take into account the so-called ideal nonlinearity of deformation, allowing to obtain some additional opportunities for economical design of building constructions. At the same time, they do not give an opportunity, unfortunately, to investigate the stress-strain state of reinforced concrete constructions in the operational stage under time-varying external load regimes, as well as to reveal the peculiarities of the redistribution of internal forces from the more loaded sections and the cross-section components to less loaded ones.

Modern norms and design practice allow for the consideration of rheology and nonlinearity of deformation at the recommendatory level and mainly for static calculations for planar and three-dimensional structures. Although there are numerous methods and proposals that allow taking into account these factors, it is true, basically, again for static loading conditions.

The current methodology for assessing the adaptability and redistribution of forces under dynamic effects on reinforced concrete constructions is inaccurate and, in fact, empirical. It does not allow us to seek a complex solution of the problem, taking into account the nonlinearity and duration of deformation, the regime and duration of loading, the features of energy absorption, vibro-creep, etc. and their influence on the process of redistribution of efforts.

To answer the above questions, the integral estimation method allows. In this case, it is necessary to consider the following prerequisites as valid: the geometric scheme of the constructions does not change even at the moment immediately preceding the failure. This is due to the fact that at that moment the load-bearing capacity of the cross-sections exists, with at least a minimal, but still finite rigidity. And in dangerous sections, the dangerous development of forces under the influence of an increasing external load is inhibited by the process of redistribution of forces and reaches its maximum at the time of destruction; the assumption of smallness of deformations with a finite value of displacements remains; the hypothesis on the additivity of causes and the consequences of deformation, which is otherwise called the principle of the independence of the action of forces, is considered fair. The mechanical interpretation of the law of conservation of energy in modern mechanics of a deformable solid body, expressed by the principle of possible displacements, which asserts the equality of the work of external and internal forces on every possible infinitesimal displacement of points of the system, is applicable both for ideally elastic and for nonlinearly, non-equilibrium and anisotropic- deformable systems. The application of this principle to perfectly elastic systems assumes that the entire work of internal forces is spent only on the formation of deformations and accumulates in the form of potential energy. Its use in systems with imperfections of deformation requires taking into account possible irreversible energy consumption for hysteresis losses, residual deformation, overcoming of internal friction and heat generation.

In connection with the fact that the energy expenditure during the deformation of real bodies depends both on the regimes and the duration of the action of external loads, and on the level of internal forces formed, then in the energy balance equations the irreversible energy losses mentioned above, must be taken into account, in addition, and equations of equilibrium and energy balance equations must be written for an infinitesimal time interval.

The assumption of smallness of deformation is applicable to reinforced concrete constructions, since their absolute dimensions are always much greater than their absolute deformations, and this assumption is valid regardless of the type of the equation of the mechanical state of the materials used.

The additivity hypothesis, formulated in mechanics in the form of the principle of independence of the action of forces, is of fundamental importance in the methods of calculating building constructions. However, for calculation of non-linearly deformable 
systems it is, strictly speaking, not applicable, but for linear but nonequilibrium-deformable systems it is suitable only for synchronous changes in time of external loads and mechanical properties of materials. At the same time, the additivity hypothesis is not limited to the principle of the independence of the action of forces, it can be interpreted as a feature of the mathematical form of recording the resolving equations in displacements or in stresses (15).

If we assume that the displacements of the system must reflect the overall level of the stress-strain state of the system, then they become dependent on this level of the stressed state, but the form of recording the equations does not change. However, the implementation of such a mathematical apparatus is possible only through the application of the method of integral estimates in combination with a system of successive approximations that clarifies the stress state with allowance for all kinds of redistribution of forces and stresses in the cross sections. The hypothesis of additivity is expedient to use only when the linearizing sense of the integral estimator method is taken into account.

\section{Conclusions}

In the method of integral estimates, process of successive approximations is not just a mathematical technique used instead of closed solution, but a method of integral refinement, based on an iterative process, internal forces and stresses, transforming over time due to their redistribution from more stressed sections and components of the cross sections on the less loaded.

Following the adoption of such an assumption, derived above, integral-differential equations apply to non-linear differential permitting decisions based on the finite difference method or finite element, which in turn transforms them into a system of nonlinear algebraic equations.

Under these conditions, it is necessary to take into account that the coefficients of the system of algebraic equations themselves become nonlinear and depend on the level of the stress state and require constant refinement in the course of the solution based on the method of successive approximations. It is necessary to use special techniques that improve the convergence of the iterative process.

Considering the process of dynamic deformation of an elastically creeping body, it is possible to construct a calculation technique under dynamic load conditions of any type, and the case of harmonic effects will be the simplest.

To realize the solution of the problem in the non-linear formulation, which provides the dependence of the physic-mechanical characteristics of the materials on the level of the stressed state of the material, it is required to find the magnitude of the acting stress on each of the faces of each volumetric finite element at each stage of the iteration process. To this end, we should use the well-known dependences (14), bearing in mind that instead of the constants adopted in the theory of elasticity, we must consider nonlinear integral operators (15), (16).

It is necessary to bear in mind too one significant drawback inherent in the finite element method, which consists in the fact that the displacement field in a deformable body divided into finite elements is continuous, since for the chosen law of change of displacements for the entire system the conditions of continuity of displacements are automatically satisfied. As for the field of stresses and deformations, while remaining constant (under the linear law of displacement) in each finite element, they undergo discontinuities at the boundaries between the elements, i.e. on the lines of division of the continuum into finite elements.

Thus, the above reasoning makes it possible to find a numerical solution of the posed problem for a triaxial nonlinear and elasto-creeping deformed body under the conditions of 
the dynamic action of external loads using the method of integral estimates based on the stepping method and successive approximations. The implementation of such method in high-rise construction will more fully reflect the real properties of materials accounting their actual behavior under the influence of dynamic loads that will make it possible to save material resources.

\section{References}

1. V.M. Bondarenko, V.I. Rimshin. The theory of dissipative power resistance of reinforced concrete, 287 (2015)

2. E.M. Zverjaev, M.V. Berlinov, M.N. Berlinova. The integral method of definition of basic tension condition of anisotropic shell.International Journal of Applied Engineering Research, 11(8), 5811 (2016)

3. E.A. Korol, M.V. Berlinov, M.N. Berlinova, Kinetics of the strength of concrete in constructions.Procedia Engineering, 165, 292 (2016)

4. E.A. Korol, Yu.A. Kharkin, Book of Reports of XX Russian-Polish-Slovak Workshop "Theoretical Fundamentals of Construction" 401 (2011)

5. V.M. Bondarenko,V.I.Kolchunov. Calculation models of the power resistance of reinforced concrete, 112 (2004)

6. A.G. Tamrazyan, S. G. Yesayan. Mechanics of creep of the concrete, 490 (2012) 